$\Rightarrow$ CANCER DIAGNOSIS

\title{
Catching cancer
}

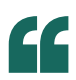

nanosensors to diagnose lung

cancer in mice by detecting and amplifying the activity of dysregulated extracellular proteases
Improved outcomes for patients with cancer are highly dependent on early detection. Currently our best form of detection is cancer screening. Yet, this has limitations. For example, in the US, patients at high risk for lung cancer are screened using lowdose computed tomography (LDCT) but this method is often associated with false-positive results. Furthermore, invasive follow-up procedures to confirm diagnosis are fraught with complications that can cause death or debilitation. As a result, there has been an impetus to develop non-invasive molecular diagnostic tests. One such means is through the detection of blood-based biomarkers, such as circulating tumour DNA. Now, the group of Sangeeta Bhatia have leveraged a set of nanosensors to diagnose lung cancer in mice by detecting and amplifying the activity of dysregulated extracellular proteases, offering a urine-based readout.

Building on their earlier work, which had shown that activitybased cancer biomarkers had the necessary diagnostic sensitivity, the authors focused here on determining the specificity of their assay using a mouse model that most closely recapitulates human

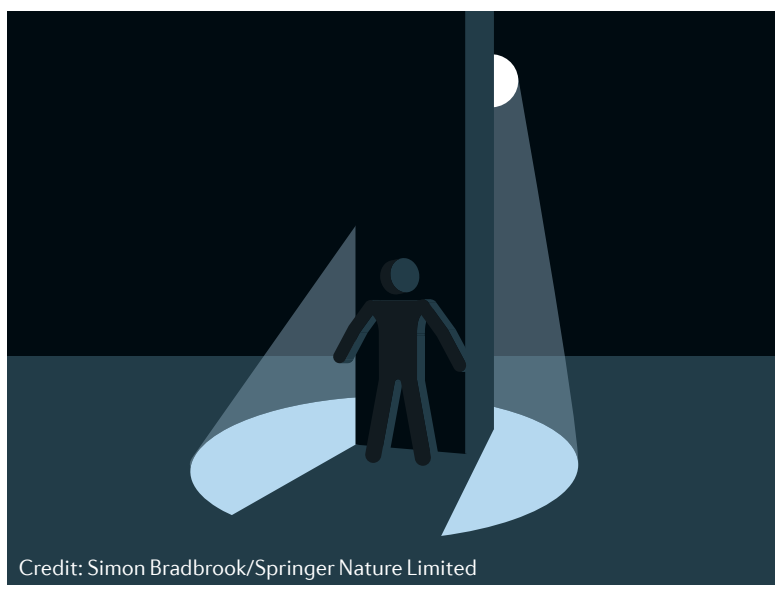

lung adenocarcinoma (LUAD), the $\mathrm{Kras}^{\mathrm{LSL}-\mathrm{G} 12 \mathrm{D} /+} ; \operatorname{Trp} 53^{\mathrm{fl} / \mathrm{fl}}(\mathrm{KP})$ mouse. The assay takes the form of generating nanoparticles coated with protease peptide substrates conjugated to mass-spectrometry-encoded reporters. Once delivered, the nanoparticles accumulate in tumours where tumour-associated proteases cleave the substrates, releasing the reporters, which in turn diffuse into the circulation, are filtered through the kidney and can then be detected in a urine sample.

Mining previously published RNA sequencing and bulk gene expression microarray datasets from mouse Kras-mutant and KP tumours enabled the authors to identify extracellular proteases that were upregulated relative to normal lung tissues. The overexpressed proteases in the KP model were then compared with those proteases upregulated in human LUAD mined from the cancer genome atlas (TCGA) dataset. Many of the proteases overlapped between KP tumours and human LUAD, justifying the use of this preclinical model to replicate human disease conditions.

To generate the nanosensor panel, 15 protease genes encoding metalloproteinases, serine proteases and aspartic proteases were selected, comprising 6-7 of the top overexpressed genes from each of the human and mouse datasets. Next, a set of 14 substrates were synthesized that would cover the cleavage preferences of the 15 selected proteases. Each substrate was then tagged to a distinct reporter, which functioned as a barcode.

The authors reasoned that administering the nanosensors via the mouse airways rather than intravenously would be the most suitable delivery system to avoid background protease activity present in the blood and off-target organs. Testing the delivery of unconjugated nanoparticles to healthy mice by intratracheal instillation revealed deep penetration of the nanoparticles to all lobes of the lung and within terminal alveoli. In the setting of a late-stage KP tumour-bearing mouse, intrapulmonary delivery of the same nanoparticles showed that they could reach the tumour periphery.

To evaluate the diagnostic accuracy of the activity-based nanosensors, KP mice were treated intratracheally with all 14 multiplexed substrate-coupled nanoparticles in a single dose at 5 , 7.5 and 10.5 weeks after tumour induction. This demonstrated that several of the reporters were differentially enriched in the urine of KP mice compared with healthy controls at 7.5 and 10.5 weeks but not at 5 weeks, enabling detection of lung cancer with $100 \%$ specificity and $81 \%$ sensitivity. Furthermore, the authors were able to establish that the assay could extend to detect other lung cancer subtypes; using the same approach in a mouse model driven by the EML4-ALK fusion revealed urinary reporter differences at all three time points, which discriminated tumour-bearing mice from healthy mice with $100 \%$ specificity and $95 \%$ sensitivity.

In validation of the specificity of their assay, it was also able to distinguish KP mice and EML4ALK-mutant mice from those with benign inflammatory disease stimulated by intratracheal delivery of lipopolysaccharide.

This study has demonstrated the translational potential of lung-specific activity-based nanosensors and suggests that for lung cancer detection, they could serve as an adjunct or alternative to LDCT.

Anna Dart

ORIGINAL ARTICLE Kirkpatrick, J. D. et al. Urinary detection of lung cancer in mice via noninvasive pulmonary protease profiling. Sci. Transl Med. 12, eaaw0262 (2020) 